\title{
riccafd
}

Revista Iberoamericana de Ciencias de la Actividad Física y el Deporte

\section{ANÁLISIS BIBLIOGRÁFICO SOBRE LOS PROGRAMAS DE RECREOS ACTIVOS}

\section{A BIBLIOGRAPHIC REVIEW OF ACTIVE RECESS PROGRAM.}

\author{
Martín-Acosta, Fátima ${ }^{1}$ y Escaravajal Rodríguez, Juan Carlos² \\ ${ }^{1}$ Martín-Acosta, Fátima. Facultad de Educación, Universidad de Cádiz, España. \\ fatima.martin@uca.es \\ ${ }^{2}$ Escaravajal Rodríguez, Juan Carlos. Facultad de Ciencias de la Educación, Universidad de \\ Granada, España. jcescaravajalrodriguez@gmail.com
}

Correspondencia: Fátima Martín-Acosta. fatima.martin@uca.es

DOI: http://dx.doi.org/10.24310/riccafd.2019.v8i1.5790

\section{RESUMEN}

El objetivo del presente trabajo consiste en revisar, recopilar y analizar la bibliografía relacionada con los programas de recreos activos en centros escolares. Se realizó un revisión documental de los artículos publicados desde 2010 hasta septiembre de 2018 en las siguientes bases de datos: Dialnet, EBSCOhost, Google Scholar y Web of Science. Las palabras clave utilizadas fueron: recreos activos, active recreation y active recess. De esta búsqueda se obtuvieron un total de 153 artículos, publicados entre 2010 y 2018, posteriormente se llevó a cabo un proceso de selección final aplicando los criterios de inclusión: (1) artículos originales; (2) publicados en revistas indexadas; (3) escritos en castellano o inglés; y (4) artículos que describen las actuaciones desarrolladas durante los recreos. Obteniendo finalmente 10 artículos para analizar. En los resultados se observó, en relación a la tipología, que los más presentes son de experiencia $(50 \%)$, seguidos de investigación (40\%). Por otro lado y en relación a las etapas en las que están enfocados, Primaria es la más representada con un $56,3 \%$, seguida de Infantil (25\%). En base a los programas analizados, se observa que las actuaciones más empleadas para fomentar la práctica de actividad física en los recreos son: organización de torneos, habilitación de nuevos espacios deportivos, decoración del patio, realización de juegos cooperativos, adquisición de nuevo equipamiento deportivo y fabricación de materiales autoconstruidos.

Palabras clave: actividad física, sedentarismo, convivencia, horario escolar, recreación. 


\section{ABSTRACT}

The aim of this project is reviewing, compiling and analyzing the bibliography related to the active recess programs in school centers. A documental review was conducted, containing all the published articles from 2010 to 2018 in the following databases: Dialnet, EBSCOhost, Google Scholar and Web of Science. The keywords used were: recreos activos, active recreation and active recess. From this search, 153 articles were found, and after that a selection was made with the following deferral criteria: (1) to be original articles; (2) published in indexed journals; (3) articles written in spanish or english; and (4) articles that describe the development of the activities during the recess. A total of 10 articles were obtained for the analysis. In the results, it was observed that the most common were those of experiences $(50 \%)$, followed by researches (40\%). Related to the educational stages aimed for, Primary was the most common (56,3\%), followed by Preschool $(25 \%)$. According to the analyzed programs, it is observed that the most usual actions to encourage the physical activity in recesses are: tournaments, cooperative games, adquisition of new sport gear and the building of self-constructed materials.

Key words: physical activity; sedentary lifestyle; coexistence; school schedule; recreation.

\section{INTRODUCCIÓN}

Los bajos niveles de actividad física (AF) en la población infantojuvenil se convierte en una situación preocupante (Riddoch et al., 2007). La Organización Mundial de la Salud (OMS, 2010), debido a sus claros beneficios hacia la salud, recomienda la realización de 60 minutos de AF moderada o vigorosa al día y un mínimo de 3 veces por semana la ejecución de actividades de fortalecimiento del sistema musculoesquelético.

En este sentido, la escuela juega un papel importante en la promoción de AF, ya sea en Educación Física (EF), con la oferta de actividades extraescolares o con el fomento de los recreo activos, siendo la última una forma factible para acometer el objetivo de aumentar la AF entre nuestro alumnado (Méndez-Giménez, 2016). Se ha demostrado que el recreo puede contribuir hasta un $40 \%$ de los 60 minutos diarios recomendados de AF (Frago-Calvo, Murillo, García-González, Aibar y Zaragoza, 2017). Además, la investigación de Pate y O’Neill (2008) sugiere que, en condiciones apropiadas, el entrono escolar puede desempeñar un papel crucial en la promoción de la AF y estilos de vida saludables.

Diversas investigaciones han determinado que los niveles de AF realizada en los recreos no contribuyen a lograr las recomendaciones de la OMS (Álvarez, Villalobos y Vargas, 2018; Martínez, Aznar y Contreras, 2015), así como las barreras que los alumnos se encuentran, en las que se identifican: la falta de instalaciones y equipos, la falta de apoyo por parte de los docentes y la falta de espacio, entre otros (Álvarez, Villalobos y Vargas, 2018). 
En esta línea, se han desarrollado diferentes programas como por ejemplo: organización de actividades dirigidas, autoconstrucción de materiales, creación de zonas de jugos, dotación de equipamiento (canastas, porterías, balones, etc.), y organización de campeonatos, entre otros (Méndez-Giménez, Cecchini y Fernández-Río, 2017; Navarro, 2012; Pallasá y Méndez-Giménez, 2016), comprobando el éxito de este tipo de actuaciones en el aumento de la AF en el alumnado (Chin y Ludwig, 2013; Erwin, Ickes, Ahn, y Fedewa, 2014; Lamoneda y Huertas, 2017; Nogués, 2010). Así, Martínez et al. (2015) apoyan que los recreos deberían ser escenarios de tiempo y espacios saludables.

Por otro lado, además de la AF, la convivencia es otro elemento presente en el recreo y en el día a día durante la jornada escolar, convirtiéndose éste en un lugar donde nacen y se refuerzan las relaciones personales (Castillo, 2014), y donde surgen conflictos y disputas entre el alumnado (Burción, 2015). En este caso, en los programas de dinamización de los recreos también se ha encontrado utilidad para disminuir los conflictos y mejorar la convivencia (Castillo-Rodríguez, Picazo y Gil-Madrona, 2018).

El objetivo del presente trabajo consiste en revisar, recopilar y analizar la bibliografía relacionada con los programas de recreos activos en centros escolares y así tener un recurso que facilite el acercamiento a la trayectoria y actualidad de los programas de recreos activos.

\section{METODOLOGÍA}

\section{Diseño}

El diseño utilizado para esta investigación ha sido la revisión documental descriptiva (Hernández, Fernández y Baptista, 2010) de la bibliografía científica relacionada los recreos activos en el ámbito educativo.

\section{Procedimiento}

La búsqueda se centró en artículos publicados desde 2010 hasta septiembre de 2018, para ella se utilizaron las siguientes bases de datos: Dialnet, EBSCOhost, Google Scholar y WOS (Web of Science).

Las palabras clave utilizadas fueron: recreos activos, active recreation y active recess.

De esta búsqueda se obtuvieron un total de 153 artículos, publicados entre 2010 y 2018, posteriormente se llevó a cabo un proceso de selección final aplicando los siguientes criterios de inclusión (figura 1): (1) artículos originales; (2) publicados en revistas indexadas; (3) escritos en castellano o inglés; y (4) artículos que describen las actuaciones desarrolladas durante los recreos. 
Búsqueda en bases de datos: Dialnet, EBSCOhost, Google Scholar y WOS.

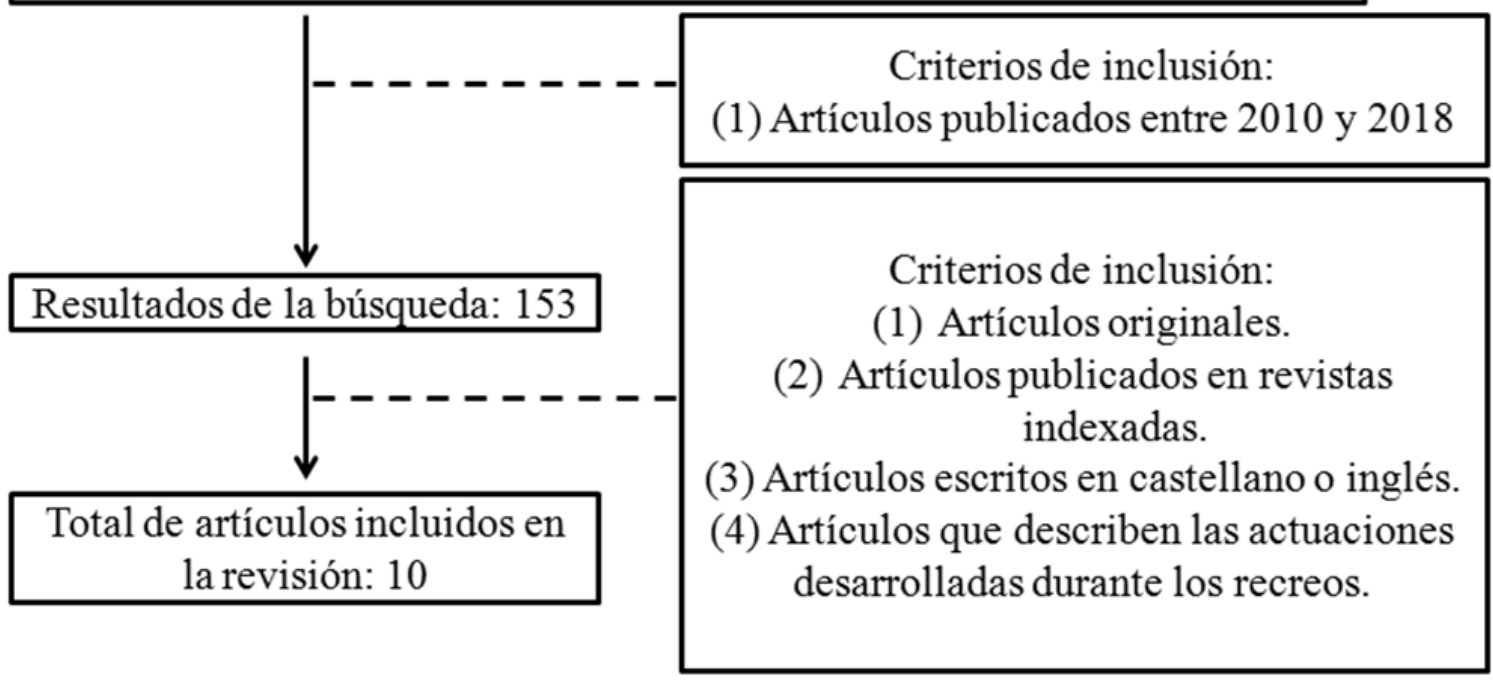

Figura 1. Proceso de selección de los artículos.

\section{Análisis estadístico}

Para el análisis de los datos obtenidos se realizó una estadística descriptiva con el software Statistical Package for Social Sciences v.20.0 (SPSS).

\section{RESULTADOS}

Atendiendo a la tipología, los artículos fueron clasificados en "propuesta", "experiencia" e "investigación". Los trabajos que más predominaron fueron los de experiencia $(50 \%)$, seguidos por los de investigación con un $40 \%$, y por último, los de propuesta con un $10 \%$. Por otro lado, y en relación a las etapas en las que están enfocados, Primaria es la más representada con un $56,3 \%$, seguida de Infantil con un $25 \%$, E.S.O. con un $12,5 \%$, y con un $6,2 \%$ Bachillerato.

De los artículos seleccionado (tabla 1) se realizó un análisis de distintas variables.

Tabla 1: Trabajos seleccionados.

\begin{tabular}{|c|c|c|c|c|}
\hline Autoría & Tipo* & Etapa & Actuaciones & Evaluación de objetivos \\
\hline $\begin{array}{c}\text { Navarro } \\
\text { (2012) }\end{array}$ & $E$ & Primaria & $\begin{array}{l}\text { - Habilitación de zonas para } \\
\text { floorball, mazaball, ultímate } \\
\text { y datchball, y organización de } \\
\text { torneos. }\end{array}$ & $\begin{array}{l}\text { Aumento AF: No } \\
\text { Participación/motivación: } \\
\text { No }\end{array}$ \\
\hline $\begin{array}{l}\text { Chin y } \\
\text { Ludwig } \\
(2013)\end{array}$ & 1 & Primaria & $\begin{array}{l}\text { - Realización de juegos coope- } \\
\text { rativos dirigidos por un coordi- } \\
\text { nador de juegos. }\end{array}$ & $\begin{array}{l}\text { Aumento AF: Si (observa- } \\
\text { ción) } \\
\text { Participación/motivación: } \\
\text { No }\end{array}$ \\
\hline
\end{tabular}


Tabla 1: Trabajos seleccionados (continuación).

\begin{tabular}{|c|c|c|c|c|}
\hline Autoría & Tipo* & Etapa & Actuaciones & Evaluación de objetivos \\
\hline $\begin{array}{l}\text { Pallasá y } \\
\text { Méndez- } \\
\text { Giménez } \\
\text { (2016) }\end{array}$ & $E$ & $\begin{array}{l}\text { Infantil y } \\
\text { Primaria }\end{array}$ & $\begin{array}{l}\text { - Habilitación de nuevos espa- } \\
\text { cios. } \\
\text { - Decoración del patio. } \\
\text { - Fabricación de materiales au- } \\
\text { toconstruidos: pelotas globo, } \\
\text { gábatons, chapas, tetrapala, } \\
\text { carioca, indiaca, fichas multi- } \\
\text { lanzamiento, duni y pelotas de } \\
\text { tetrapala. }\end{array}$ & $\begin{array}{l}\text { Aumento AF: No } \\
\text { Participación/motivación: } \\
\text { Si (observación) }\end{array}$ \\
\hline $\begin{array}{l}\text { Castillo- } \\
\text { Rodríguez } \\
\text { et al. } \\
\text { (2018) }\end{array}$ & 1 & Primaria & $\begin{array}{l}\text { - Desarrollo de actividades: } \\
\text { comba/ reloj, fútbol, partidos } \\
\text { quemaos, goma elástica, jue- } \\
\text { gos populares: rayuela y zapa- } \\
\text { tilla por detrás, carrera de sa- } \\
\text { cos y baloncesto (KAO). }\end{array}$ & $\begin{array}{l}\text { Aumento AF: No } \\
\text { Participación/motivación: } \\
\text { Si (cuestionarios y obser- } \\
\text { vación) }\end{array}$ \\
\hline $\begin{array}{l}\text { Méndez- } \\
\text { Giménez } \\
\text { y Pallasá- } \\
\text { Manteca } \\
\text { (2018) }\end{array}$ & 1 & $\begin{array}{l}\text { Infantil y } \\
\text { Primaria }\end{array}$ & $\begin{array}{l}\text { - Habilitación de nuevos espa- } \\
\text { cios. } \\
\text { - Fabricación de materiales au- } \\
\text { toconstruidos: pelotas-globo, } \\
\text { aros-ringos, pompones, gába- } \\
\text { tons, tetrapalas y pelotas, in- } \\
\text { diacas, cors para jugar al duni, } \\
\text { cariocas, chapas, fichas multi- } \\
\text { lanzamiento para jugar al cas- } \\
\text { cayu (rayuela), siete y media, } \\
\text { dianas, líneas, etc. } \\
\text { - Decoración con juegos infan- } \\
\text { tiles: cascayu, twistter, scocth- } \\
\text { scotch, cuatro cuadros, mini- } \\
\text { campos de fútbol-chapa, cir- } \\
\text { cuitos de chapas, dianas, juego } \\
\text { de las siete y media, etc. }\end{array}$ & $\begin{array}{l}\text { Aumento AF: No } \\
\text { Participación/motivación: } \\
\text { Si (cuestionarios) }\end{array}$ \\
\hline $\begin{array}{l}\text { García y } \\
\text { Serrano } \\
\text { (2010) }\end{array}$ & $E$ & $\begin{array}{l}\text { Infantil y } \\
\text { Primaria }\end{array}$ & $\begin{array}{l}\text { - Se mejoró el espacio y se creó } \\
\text { diferentes zonas de juegos. } \\
\text { - Adquisición de nuevo mate- } \\
\text { rial deportivo y didáctico. } \\
\text { - Organización del patio en zo- } \\
\text { nas por actividades: Combas, } \\
\text { zancos, fútbol, aros, voleibol, } \\
\text { bola canaria, baloncesto, el } \\
\text { brilé y aula de música. }\end{array}$ & $\begin{array}{l}\text { Aumento AF: No } \\
\text { Participación/motivación: } \\
\text { Si (informes) }\end{array}$ \\
\hline
\end{tabular}


Tabla 1: Trabajos seleccionados (continuación).

\begin{tabular}{|c|c|c|c|c|}
\hline Autoría & Tipo* & Etapa & Actuaciones & Evaluación de objetivos \\
\hline $\begin{array}{l}\text { Lizcano, } \\
\text { Martín } \\
\text { y García } \\
\text { (2018) }\end{array}$ & $E$ & $\begin{array}{l}\text { Infantil y } \\
\text { Primaria }\end{array}$ & $\begin{array}{l}\text { - Se compraron materiales: } \\
\text { pinturas para el suelo, jugue- } \\
\text { tes, cajas para guardar los ju- } \\
\text { guetes, libros, etc. } \\
\text { - Distribución de los espacios } \\
\text { mediante áreas delimitadas y } \\
\text { visibles, y se introdujeron pic- } \\
\text { togramas. Las zonas quedaron } \\
\text { delimitadas en: fútbol, juegos } \\
\text { tradicionales (sambori, rayue- } \\
\text { la, oca, pollito inglés...) y zona } \\
\text { del porche (donde se juega } \\
\text { con muñecos, juegos de mesa, } \\
\text { cuentos, conversar, etc.). }\end{array}$ & $\begin{array}{l}\text { Aumento AF: No } \\
\text { Participación/motivación: } \\
\text { Si (informes y cuestiona- } \\
\text { rios) }\end{array}$ \\
\hline $\begin{array}{l}\text { Rodríguez } \\
\text { y Arjona } \\
\text { (2012) }\end{array}$ & $\mathrm{P}$ & $\begin{array}{l}\text { Primaria } \\
\text { y Secun- } \\
\text { daria }\end{array}$ & $\begin{array}{l}\text { - Desarrollo de una liga en pri- } \\
\text { meria con las siguientes ac- } \\
\text { tividades: Mate, Voley-tenis, } \\
\text { Pichi, Fútbol } 4 \times 4 \text { y Balonmano } \\
\text { - Desarrollo de una liga en } \\
\text { E.S.O. con las siguientes activi- } \\
\text { dades: balonkorf, balón torre, } \\
\text { atletismo, balonmano, volei- } \\
\text { bol, béisbol, el quema, prue- } \\
\text { bas combinadas: sogatira y } \\
\text { relevos, y diseño coreográfico. }\end{array}$ & $\begin{array}{l}\text { Aumento AF: No } \\
\text { Participación/motivación: } \\
\text { No }\end{array}$ \\
\hline $\begin{array}{c}\text { Martínez } \\
\text { de Ojeda } \\
(2015)\end{array}$ & 1 & Primaria & $\begin{array}{l}\text { - Realización de entrenamien- } \\
\text { tos relacionados con las uni- } \\
\text { dades didácticas que se están } \\
\text { desarrollando en EF, deportes: } \\
\text { balonmano y fútbol sala. }\end{array}$ & $\begin{array}{l}\text { Aumento AF: No } \\
\text { Participación/motivación: } \\
\text { Si (observación e infor- } \\
\text { mes) }\end{array}$ \\
\hline $\begin{array}{c}\text { Nogués } \\
\text { (2010) }\end{array}$ & $E$ & $\begin{array}{l}\text { Secunda- } \\
\text { ria y Ba- } \\
\text { chillerato }\end{array}$ & $\begin{array}{l}\text { - Realización de campeonatos } \\
\text { de diferentes deportes: fútbol } \\
\text { sala, baloncesto, voleibol, ba- } \\
\text { loncesto } 3 \times 3 \text {, mini-voley, bád- } \\
\text { minton, tiros libres y triples. } \\
\text { - Exhibiciones de baile. }\end{array}$ & $\begin{array}{l}\text { Aumento AF: No } \\
\text { Participación/motivación: } \\
\text { No }\end{array}$ \\
\hline
\end{tabular}

*P: propuesta; E: experiencia; I: investigación.

\section{DISCUSIÓN}

En base a los resultados obtenidos, los programas de recreos activos estaban enfocados principalmente a Primaria e Infantil, y en menor medida a E.S.O y Bachillerato, algo positivo si tenemos en cuenta que la infancia es considerada un momento significativo para desarrollar hábitos de vida saludables (OMS, 2010). En esta línea, en los estudios de Frago-Calvo et al. (2017) y Serra (2014) se concluyó que el alumnado de primaria era más activo que el de E.S.O, existien- 
do una disminución de los niveles de AF a medida que aumenta la edad. Por lo tanto, sería conveniente darles una especial atención en cuanto a la implementación de programas de recreos activos en los institutos, siendo también escasa su presencia en la literatura científica como se observa en el presente trabajo.

Uno de los trabajos analizados (Lizcano et al., 2018) va enfocado a favorecer la inclusión del alumnado con trastorno del espectro autista (TEA), habiendo ya programas de inclusión en los que se han encontrados resultados positivos con respecto a la mejora de interacción social en el alumnado con necesidades especiales (D'Angelo, 2017). El recreo es uno de los momentos de la jornada escolar donde los alumnos pueden relacionarse libremente, por lo que es un espacio ideal donde trabajar en la diversidad del alumnado para facilitar la interacción con sus iguales (Artiles, Marchena y Santana, 2018).

En relación a las actividades desarrolladas en el tiempo del recreo, se ha obtenido que los niños suelen realizar actividades de equipo como el fútbol, baloncesto o balonmano, en cambio, las niñas normalmente dan paseos por el patio (Escalante, Backx, Saavedra, García-Hermoso y Domínguez, 2011; García, 2018). Por ello, a través de los programas de recreos activos, para conseguir una mayor participación activa del alumnado, se fomenta la práctica de una variedad de actividades como deportes alternativos (Navarro, 2012), utilización de materiales autoconstruidos (Méndez-Giménez y Pallasá-Manteca, 2018; Pallasá y Méndez-Giménez, 2016), juegos cooperativos (Chin y Ludwig, 2013) y torneos organizados (Nogués, 2010; Rodríguez y Arjona, 2012).

Diversos estudios han definido como una de las principales barreras, hacia la práctica de AF en los recreos, el escaso equipamiento deportivo y las instalaciones de juego (Escalante, García-Hermoso, Backx y Saavedra, 2014; Lopes, Lopes y Pereira, 2009; Martínez-Andrés et al., 2015; Woods, McLoughlin, Kern y Graber, 2018). En varios de los programas de recreos activos, analizados en el presente trabajo, se observan actuaciones referentes a los espacios y materiales como: habilitación de nuevos espacios de práctica deportiva (Navarro, 2012; Pallasá y Méndez-Giménez (2016), decoración del patio (Méndez-Giménez y Pallasá-Manteca, 2018; Pallasá y Méndez-Giménez, 2016), y la adquisición de nuevos materiales (García y Serrano, 2010; Lizcano et al., 2018). En esta última actuación, la fabricación de materiales autoconstruidos es una opción factible para aumentar los niveles de AF (Méndez-Giménez y Pallasá-Manteca, 2018; Pallasá y Méndez-Giménez, 2016), siendo además una estrategia con un bajo coste económico (Méndez-Giménez et al., 2017).

Por último destacar que la forma de evaluación de la participación y motivación del alumnado, no realizada en todos los centros, fue mediante principalmente subjetiva mediante observación e informes realizados por el profesorado y cuestionarios al alumnado. Además, las investigaciones sobre los programas de AF en recreos afirman que los programas bien orientados y estructurados constituyen al aumento de los niveles de AF y mejora de la condición físicas del alumnado (Pumar, Navarro y Basanta, 2015). Sin embargo, la forma de evaluar 
este aspecto en los artículos seleccionados solo se ha dado en uno de los trabajos (Chin y Ludwig, 2013) y fue obtenido mediante la observación, por lo que sería conveniente para futuros trabajos realizar una medición más objetiva, como podría ser la acelerometría, como ya es usado en estudios similares (Godard et al., 2016).

\section{CONCLUSIONES}

Este trabajo presenta una recopilación de los artículos existentes desde 2010 hasta la actualidad en diversas bases de datos, dónde se desarrollan gran variedad de programas de recreos activos para fomentar la práctica de AF en dicho espacio. Los resultados muestran como la mayoría de programas van enfocados a edades tempranas (Infantil y Primaria), a pesar de que en investigaciones existentes se demuestra como los niveles de AF disminuyen conforme aumenta la edad, por lo que sería positivo desarrollar más programas de recreos activos en adolescentes. Por otro lado, las actuaciones que se utilizan para tal fin son: organización de torneos, habilitación de nuevos espacios deportivos, decoración del patio, realización de juegos cooperativos, adquisición de nuevo equipamiento deportivo y fabricación de materiales autoconstruidos, y desarrollo de deportes alternativos y juegos tradicionales. Además, la metodología de evaluación subjetiva de consecución de objetivos es la predominante y sería enriquecida por una evaluación más objetiva, como acelerometría.

Por tanto, se concluye que la recopilación de artículos en este trabajo, lo convierte en un útil recurso para docentes e investigadores con interés en esta temática, ya que pueden acceder de forma sencilla a todos los artículos existentes hasta el momento y obtener información práctica de ellos para desarrollarla en sus propios centros escolares.

\section{REFERENCIAS BIBLIOGRÁFICAS}

Álvarez, C., Villalobos, G., y Vargas, J. (2018). Determinación de la actividad física en el recreo escolar: Combinando mediciones de actividad física y la perspectiva estudiantil. MHSalud: Movimiento Humano y Salud, 14(2), 44-58. Doi: https://doi. org/10.15359/mhs.14-2.4.

Artiles, J., Marchena, R., y Santana, R. (2018). Los recreos del alumnado con discapacidad en centros de Educación Secundaria. Siglo Cero, 47(2), 79-98. Doi: http://dx.doi. org/10.14201/scero20164727998.

Burción, S. (2015). El recreo libre y el recreo dirigido. Comparación de conductas del alumnado para la mejora de la convivencia (Trabajo Fin de Grado). Universidad de Valladolid, Valladolid, España. Recuperado de http://uvadoc.uva.es/handle/10324/10647.

Castillo, M. N. (2014). El recreo un espacio de y para la socialización (Trabajo Fin de Grado). Universidad de Zaragoza, Zaragoza, España. Recuperado de http://zaguan. unizar.es/record/14411/files/TAZ-TFG-2014-572.pdf. 
Castillo-Rodríguez, G., Picazo, C. C., y Gil-Madrona, P. (2018). Dinamización del recreo como resolución de conflictos y participación en actividades físico-deportivas. Revista Electrónica Educare (Educare Electronic Journal), 22(2), 1-22. Doi: http://dx.doi. org/10.15359/ree.22-2.14\%0D.

Chin, J. J., y Ludwig, D. (2013). Cómo aumentar la actividad física de los niños durante el período del recreo en las escuelas. Revista Panamericana de Salud Pública, 34(5), 367-374. Doi: https://dx.doi.org/10.2105\%2FAJPH.2012.301132s.

D’Angelo, L. (2017). Proyecto Patios Divertidos: Programa de inclusión escolar para el alumnado con dificultades de interacción social en los recreos. Educ@ción en Contexto, 3(extra0), 181-210. Recuperado de https://dialnet.unirioja.es/servlet/ articulo?codigo $=6296623$.

Erwin, H. E., Ickes, M., Ahn, S., y Fedewa, A. (2014). Impact of recess interventions on children's physical activity: A meta-analysis. American Journal of Health Promotion, 28(3), 159-167. Doi: https://doi.org/10.4278\%2Fajhp.120926-LIT-470.

Escalante, Y., Backx, K., Saavedra, J. M., García-Hermoso, A., y Domínguez, A. M. (2011). Relación entre actividad física diaria, actividad física en el patio escolar, edad y sexo en escolares de educación primaria. Revista Española de Salud Pública, 85(5), 481-489. Recuperado de http://scielo.isciii.es/pdf/resp/v85n5/07_original_breve1.pdf.

Escalante, Y., García-Hermoso, A., Backx, K., y Saavedra, J. M. (2014). Playground designs to increase physical activity levels during school recess: A systematic review. Health Education \& Behavior, 41(2), 138-144. Doi: https://doi. org/10.1177\%2F1090198113490725.

Frago-Calvo, J. M., Murillo, B., García-González, L., Aibar, A. y Zaragoza, J. (2017). Physical Activity Levels during unstructured recess in spanish primary and secondary schools. European Journal of Human Movement, (38), 40-52. Recuperado de http:// www.eurjhm.com/index.php/eurjhm/article/view/410.

García, S. (2018). Valoración de la actividad física y actividad de dar paseos durante el recreo de los alumnos de primaria. Trances, 10(2), 153-168. Recuperado de http:// www.trances.es/papers/TCS\%2010_2_3.pdf.

García, M., y Serrano, J. M. (2010). La optimización del patio de recreo para favorecer la práctica de actividades físico-deportiva mediante un proyecto de convivencia. Espiral. Cuadernos del Profesorado, 3(6), 32-42. Doi: http://dx.doi.org/10.25115/ecp.v3i6.

Godard, C., Carlin, L., Torres, J., Rodríguez, M. P., Leyton, B., y Salazar, G. (2016). Nivel de intensidad de las principales actividades físicas de escolares chilenos: determinación por acelerometría. Revista chilena de nutrición, 43(3), 247-254. Recuperado de https://scielo.conicyt.cl/pdf/rchnut/v43n3/art03.pdf.

Hernández, R., Fernández, C., y Baptista, P. (2010). Metodología de la investigación. Quinta edición. México: Mcgraw-Hill Interamericana Editores. Recuperado de https:// www.esup.edu.pe/descargas/dep_investigacion/Metodologia $\% 20 \mathrm{de} \% 20 \mathrm{la} \% 20$ investigaci\%C3\%B3n\%205ta\%20Edici\%C3\%B3n.pdf.

Lamoneda, J., y Huertas, F. J. (2017). Análisis de la práctica deportiva-recreativa a través de un programa de promoción en el recreo en función del sexo en adolescentes españoles. Retos. Nuevas Tendencias en Educación Física, Deporte y Recreación, (32), 25-29. Recuperado de https://recyt.fecyt.es/index.php/retos/article/ view/49283. 
Lizcano, L., Martín, D., y García, C. (2018). La organización del recreo en alumnado con TEA: Un proyecto de patios activos. Tándem: Didáctica de la Educación Física, (62), 58-64. Recuperado de http://www.grao.com/ca/producte/la-organizacion-del-recreoen-alumnado-con-tea-ta06283268.

Lopes, L., Lopes, V., y Pereira, B. (2009). Physical activity levels in normal weight and overweight Portuguese children: An intervention study during an elementary school recess. International Electronic Journal of Health Education, 12(1), 175-184. Recuperado de https://bibliotecadigital.ipb.pt/bitstream/10198/2688/1//nternational\%20 Electronic\%20Journal\%20of\%20Health\%20Education.pdf.

Martínez, J., Aznar, S., y Contreras, O. (2015). El recreo escolar como oportunidad de espacio y tiempo saludable. Revista Internacional de Medicina y Ciencias de la Actividad Física y el Deporte, 15(59), 419-432. Doi: http://dx.doi.org/10.15366/rimcafd2015.59.002.

Martínez-Andrés, M., Rodriguez-Martín, B., Bartolomé-Gutierrez, R., Pardo-Guijarro, M. J., Cañete, J., y Cavero-Redondo, I. (2015). Factores que influyen en la práctica de actividad física durante el recreo, un estudio cualitativo. Revista Andaluza de Medicina del Deporte, 8(1), 32-32. Doi: http://dx.doi.org/10.1016/j.ramd.2014.10.030.

Martínez de Ojeda, D. (2015). Participación del alumnado en la actividad física den el tiempo de recreo en función de la metodología empleada en la clase de Educación Física. Revista Actividad Física y Deporte: Ciencia y Profesión, (22), 15-27. Recuperado de http://colefcafecv.com/wp-content/uploads/2015/10/LIBRO-ED-FISICA-22.pdf.

Méndez-Giménez, A. (2016). Centros escolares promotores de actividad física. Tándem. Didáctica de la Educación Física, (52), 4-6. Recuperado de http://www.grao. $\mathrm{com} / \mathrm{ca} /$ producte/centros-escolares-promotores-de-actividad-fisica.

Méndez-Giménez, A., Cecchini, J. A., y Fernández-Río, J. (2017). Efecto del material autoconstruido en la actividad física de los niños durante el recreo. Revista de Saúde Publica, 51(58), 1-7. Doi: https://doi.org/10.1590/S1518-8787.2017051006659.

Méndez-Giménez, A., y Pallasá-Manteca, M. (2018). Disfrute y motivación en un programa de recreos activos. Apunts: Educación Física y Deportes, (134), 55-68. Doi: http://dx.doi.org/10.5672/apunts.2014-0983.es.

Navarro, A. (2012). Actividad física en el recreo: una buena oportunidad para educar. Forum Aragón: revista digital de FEAE-Aragón sobre organización y gestión educativa, (5), 42-45. Recuperado de https://dialnet.unirioja.es/descarga/articulo/3932816.pdf.

Nogués, R. (2010). Utilización de la" hora del recreo" en los centros escolares para la práctica de actividades físico-deportivas. Revista Actividad Física y Deporte: Ciencia y Profesión, (12), 47-55. Recuperado de http://colefcafecv.com/wp-content/ uploads/2013/04/Revista-colef-12.pdf.

OMS. (2010). Recomendaciones mundiales sobre la actividad física para la salud. Geneva, Switzerland: WHO. Recuperado de https://www.who.int/dietphysicalactivity/ publications/9789241599979/es/.

Pallasá, M., y Méndez-Giménez, A. (2016). Cómo incentivar la actividad física en los recreos. Tándem: Didáctica de la Educación Física, (52), 34-39. Recuperado de http:// www.grao.com/ca/producte/como-incentivar-la-actividad-fisica-en-los-recreos.

Pate, R. R., y O'neill, J. R. (2008). Summary of the American Heart Association scientific statement: promoting physical activity in children and youth: a leadership 
role for schools. Journal of cardiovascular nursing, 23(1), 44-49. Doi: https://doi. org/10.1097/01.JCN.0000305056.96247.bb.

Pumar, B., Navarro, R., y Basanta, S. (2015). Efectos de un programa de actividad física en escolares. Educación Física y Ciencia, 17(2). Recuperado de http://www.efyc. fahce.unlp.edu.ar/article/view/EFyCv17n02a01/.

Riddoch, C. J., Mattocks, C., Deere, K., Saunders, J., Kirkby, J., Tilling, K., Leary, S. D., Blair, S. N., y Ness, A. R. (2007). Objective measurement of levels and patterns of physical activity. Archives of Disease in Childhood, 92(11), 963-969. Doi: http:// dx.doi.org/10.1136/adc.2006.112136.

Rodríguez, A. G., y Arjona, J. (2012). Los recreos activos en Educación Física. Lecturas: Educación Física y Deportes, 17(170), 1-17. Recuperado de http://www.efdeportes. com/efd170/los-recreos-activos-en-educacion-fisica.htm.

Serra, J. R. (2014). Análisis de la práctica de actividad física durante el recreo escolar en adolescentes de la provincia de Huesca. Archivos de Medicina del Deporte, 31(4), 257-262. Recuperado de http://femede.es/documentos/or04_162.pdf.

Woods, A. M., McLoughlin, G. M., Kern, B. D., y Graber, K. C. (2018). What's Physical Activity Got to Do With It? Social Trends in Less Active Students at Recess. Journal of School Health, 88(7), 500-507. Doi: https://doi.org/10.1111/josh.12637. 